\title{
Performance Analysis of MRT-Based Dual-Polarized Massive MI MO System with Space-Polarization Division Multiple Access
}

\author{
Jun-Ki Hong \\ Department of Electrical and Electronic Engineering, Youngsan University \\ Yangsan-si, Gyeongsangnam-do 50510 - South Korea \\ [e-mail: jkhong@ysu.ac.kr] \\ *Corresponding author: Jun-Ki Hong
}

Received February 28, 2018; revised May 31, 2018; accepted June 10, 2018; published August 31, 2018

\begin{abstract}
In recent years, one of the most remarkable $5 \mathrm{G}$ technologies is massive multiple-input and multiple-output (MIMO) system which increases spectral efficiency by deploying a large number of transmit-antennas (eg. tens or hundreds transmit-antennas) at base station (BS). However, conventional massive MIMO system using single-polarized (SP) transmit-antennas increases the size of the transmit-array proportionally as the number of transmit-antennas increases. Hence, size reduction of large-scale transmit-array is one of the major concerns of massive MIMO system.

To reduce the size of the transmit-array at BS, dual-polarized (DP) transmit-antenna can be the solution to halve the size of the transmit-array since one collocated DP transmit-antenna deploys vertical and horizontal transmit-antennas compared to SP transmit-antennas. Moreover, proposed DP massive MIMO system increases the spectral efficiency by not only in the space domain but also in the polarization domain whereas the conventional SP massive MIMO system increases the spectral efficiency by space domain only.

In this paper, the comparative performance of DP and SP massive MIMO systems is analyzed by space division multiple access (SDMA) and space-polarization division multiple access (SPDMA) respectively. To analyze the performance of DP and SP massive MIMO systems, DP and SP spatial channel models (SCMs) are proposed which consider depolarized propagation channels between transmitter and receiver. The simulation results show that the performance of proposed 32 transmitter (Tx) DP massive MIMO system improves the spectral efficiency by about $91 \%$ for a large number of user equipments (UEs) compare to 32Tx SP massive MIMO system for identical size of the transmit-array.
\end{abstract}

Keywords: Dual-polarized antenna, massive MIMO, space-polarization division multiple access, XPD.

A preliminary version of this paper was presented at ICONI 2017, and was selected as an outstanding paper. 


\section{Introduction}

Global mobile data traffic will increase 24.3 exabytes by 2019 since internet connections such as cloud computing, internet of things (IoT) and entertainment content on mobile devices generate tremendous amounts of data [1-3]. To handle today's massive amounts of data traffic, femtocell and massive multiple-input and multiple-output (MIMO) technologies are mainly considered to catch up with data traffic demand with high spectral efficiency.

The basic concept of a femtocell is to increase the spectral efficiency with a small base station that covers a small area by reducing the distance between the transmitters and the receivers [4][5]. Another key technology of 5G wireless communications is a massive MIMO system consisting of tens or hundreds of transmit-antennas that transmitting signals to tens or even hundreds of user devices (UEs) at the same time [6][7]. A large number of transmit-antennas at base station (BS) remarkably improves the performance by increasing multiplexing order with high degree of freedoms [8]. However, a large size of transmit-array of massive MIMO system is one of the major problems since the size of the transmit-array increases proportionally as the number of transmit-antennas increases without limitations. Therefore, transmit-antenna must be placed effectively to avoid an unlimited increase in the size of the transmit-array.

Since one collocated dual-polarized (DP) transmit-antenna composed with vertical and horizontal transmit-antennas, two spatially separated single-polarized (SP) transmit-antennas can be replaced by one DP transmit-antenna to avoid an unlimited increase in transmit-array size. Therefore, the size of the SP transmit-array can be reduced in half by the DP transmit-array.

In this paper, massive MIMO system with DP transmit-array called DP massive MIMO system is proposed to install twice as many transmit-antennas in the same space as SP massive MIMO system. To the best of the author's knowledge, the comparative performance analysis between DP and SP massive MIMO systems by system-level simulation is not available yet. To evaluate the performance of DP and SP massive MIMO systems, DP and SP MIMO spatial channel models (SCMs) are proposed which consider depolarized propagation channels with cross polarization discrimination (XPD). Then, comparative performance of DP and SP massive MIMO systems is analyzed by space-polarization division multiple access (SPDMA) and space division multiple access (SDMA) respectively via system-level simulation.

The remainder of this paper is organized as follows. In Chapter 2, related work of massive MIMO system is described. Chapter 3 describes proposed DP massive MIMO system. Chapter 4 describes SPDMA. Chapter 5 describes proposed DP and SP MIMO SCMs. Chapter 6 describes the evaluation method of DP and SP massive MIMO systems. Finally, the performance analysis of DP and SP massive MIMO systems is presented in Chapter 7 before conclusion in Chapter 8.

\section{Related Work}

During the past decades, research on MIMO system has been actively conducted to increase performance. Recently, massive MIMO system is introduced to increase performance by an extremely large number of transmit-antennas at BS. However, there are some problems with massive MIMO systems although a large number of transmit-antennas provide more spectral efficiency than conventional MIMO systems. One of the major problems of massive MIMO 
system is that the size of the transmit-array at BS increases in proportion to the number of transmit-antennas. Therefore, deployment of a large number of transmit-antennas in limited space is very important to realize practical massive MIMO system.

To deploy a large number of transmit-antennas in limited space, previously 2D planar type of transmit-array has been introduced [9][10]. The basic idea of the 2D planar MIMO system is that transmit-antennas installed on the 2D planar array instead of a 1D linear array. However, the 2D planar array performs lower than 1D linear array since the higher correlation is generated among surrounding transmit-antennas of 2D planar array compared to 1D linear array. In [11], pilot contamination of DP massive MIMO system is analyzed. However, performance analysis between DP and SP massive MIMO system by system-level simulation has not been analyzed yet.

\section{Proposed Dual-Polarized Massive MIMO System}

In this chapter, concept and advantages of proposed DP massive MIMO system are described compared with the conventional SP massive MIMO system.

\subsection{Size Reduction of Transmit-Array at BS}

Proposed DP massive MIMO system is very effective solution to reduce the size of transmit-array by deploying DP transmit-antennas which composed with two orthogonal transmit-antennas in same space. For example, 64Tx SP transmit-array with a length of $3.67 \mathrm{~m}$ can be replaced by 32Tx DP transmit-array with a length of $1.84 \mathrm{~m}$ at $1.9 \mathrm{GHz}$ center frequency with half wavelength. Therefore, the size of conventional SP transmit-array can be reduced by half by using DP transmit-array at BS. In other words, DP transmit-antenna doubles the transmit diversity compared to conventional SP transmit-antenna for same size of the transmit-array.

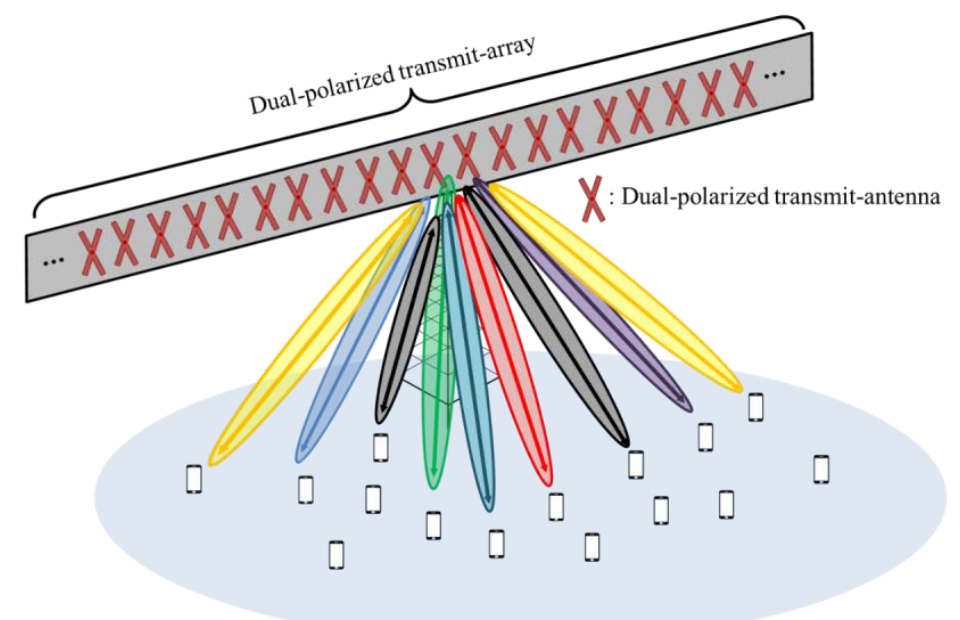

Fig. 1. Configuration of DP massive MIMO system

\subsection{Utilization in Space and Polarization Domains}

While conventional SP transmit-array utilizes the space domain via spatially separated SP transmit-antennas, DP transmit-array utilizes both space and polarization domains by spatially 
separated DP transmit-antennas and two orthogonal transmit-antennas of the collocated DP transmit-antennas. In other words, the additional utilization of polarization domain is achieved by transmitting two data streams by vertical and horizontal transmit-antennas of the collocated DP transmit-antennas.

Previously, several researches have shown that DP transmit-antenna improve performance by utilizing polarization domain with size reduction of transmit-array [9-14]. Additionally, once the SP receive-antenna receives signals in one direction, performance degrades due to polarization-mismatches [15]. Therefore, DP receive-antenna could be the solution to minimize the performance degradations from polarization-mismatches since DP receive-antenna provides wide range of receiving signals compared to SP receive-antenna. Consequently, it is clear that DP transmit and receive antennas can be the potential antenna structure for massive MIMO system to reduce the size of the transmit-array while minimizing performance degradation due to polarization-mismatches.

\section{Space-Polarization Division Multiple Access}

Proposed DP massive MIMO system increase diversity by utilizing space and polarization domains via SPDMA. On the contrary, SP transmit-array and one collocated DP transmit-antenna increase performance by SDMA and polarization division multiple access (PDMA) respectively. In this chapter, the comparative descriptions of SDMA, PDMA, and SPDMA is presented.

\subsection{Space Division Multiple Access}

SP MIMO system with SDMA increases transmit diversity by spatially separated SP transmit-antennas. However, disadvantage of SP MIMO system using SDMA is that the size of the transmit-array increases proportionally as the number of transmit-antennas increases. This is one of the major problems of the massive MIMO system. Another disadvantage is that SDMA lack of utilizing polarization domain since SDMA increases transmit diversity via space domain only.

\subsection{Polarization Division Multiple Access}

PDMA has been introduced to increase diversities via polarization domain. The basic idea of PDMA is that collocated DP transmit-antenna transmits two independent data streams to different UEs simultaneously by using vertical and horizontal transmit-antennas. The performance between a DP transmit-antenna and the DP receive-antennas with PDMA is analyzed in [13]. However, PDMA lack of utilizing space domain since only one collocated DP transmit-antenna is deployed at BS.

\subsection{Space-Polarization Division Multiple Access}

Since SDMA and PDMA lack of utilizing polarization and space domains respectively, SPDMA is introduced to utilize both space and polarization domains for DP MIMO system [14]. For instance, space and polarization diversities are achieved by spatially separated DP transmit-antennas and two orthogonal transmit-antennas of the collocated DP transmit-antennas respectively. Therefore, SPDMA increases spectral efficiency by both space and polarization domains.

Previously, comparative performance of DP and SP MIMO system is analyzed by SDMA and SPDMA respectively in [14]. However, previous SPDMA analysis lack of analyzing the 
performance of massive MIMO system with large-scale users. Therefore, in this paper, the comparative performance of DP and SP massive MIMO systems with large-scale user is analyzed by SPDMA and SDMA respectively.

\section{Proposed Dual- and Single-Polarized MIMO SCM}

3rd Generation Partnership Project (3GPP) MIMO SCM is geometry-based stochastic model which describes the excess delay, direction of arrival, and direction of departure of multipath for MIMO channel model [16]. However, conventional 3GPP MIMO SCM provides a DP MIMO SCM which considers mixed polarization effect at the DP receive-antenna. Therefore, conventional 3GPP DP MIMO SCM lack of implementing polarization diversities from depolarized propagation channels. Therefore, a DP MIMO SCM is proposed which considers depolarized propagation channels between DP transmit- and receive-antennas with cross polarization discrimination (XPD) effects.

\subsection{Dual-Polarized MIMO SCM}

As shown in Fig. 2, proposed DP MIMO SCM generate channel with four depolarized propagation channels between transmit- and receive-antennas which represented by vertical to vertical (VV), vertical to horizontal (HV), horizontal to vertical (VH), and horizontal to horizontal $(\mathrm{HH})$ polarization channels.

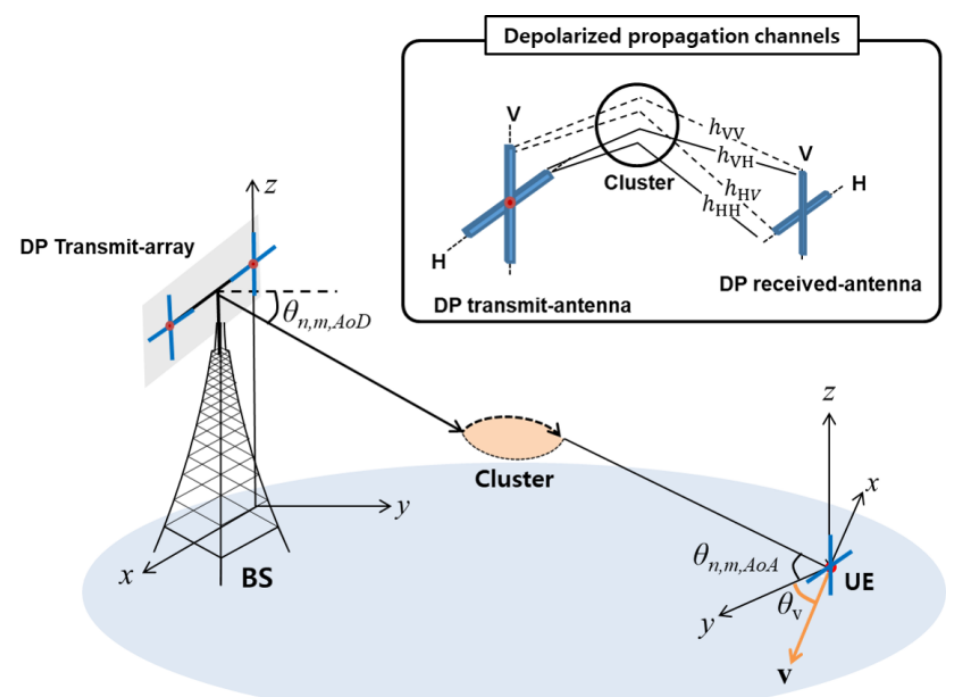

Fig. 2. Configuration of proposed DP MIMO SCM with four depolarized propagation channels

Proposed DP MIMO SCM represents BS with $S$ transmit-antennas $(S / 2 \in N$ of DP transmit-antennas) UE with $U$ receive-antennas ( $U / 2 \in N$ of DP receive-antennas). Then, $(u, s)$-th DP MIMO channel component of $n$-th multipath at time $t, \mathbf{H}_{u, s, n}(t)$, can be expressed as

$$
\mathbf{H}_{u, s, n}(t)=\sqrt{\frac{P_{n} \sigma_{S F}}{M}} \sum_{m=1}^{M}\left(\left[\begin{array}{cc}
\cos \theta & -\sin \theta \\
\sin \theta & \cos \theta
\end{array}\right]\left[\begin{array}{cc}
h_{V V} & h_{V H} \\
h_{H V} & h_{H H}
\end{array}\right] \times \exp \left(\beta_{\text {AoD }}\right) \times \exp \left(\beta_{A \circ A}\right) \times \exp \left(\psi_{\text {AoA }}\right)\right)
$$


where

$$
\begin{aligned}
& h_{V V}=\chi_{\mathrm{BS}, V}\left(\theta_{n, m, A o D}\right)\left(\sqrt{\frac{\mathrm{XPD}}{1+\mathrm{XPD}}} \exp \left(j \Phi_{n, m}^{(v, v)}\right)\right) \chi_{M S, V}\left(\theta_{n, m, A O A}\right) \\
& h_{V H}=\chi_{\mathrm{BS}, H}\left(\theta_{n, m, A o D}\right)\left(\sqrt{\frac{1}{1+\mathrm{XPD}}} \exp \left(j \Phi_{n, m}^{(v, h)}\right)\right) \chi_{\mathrm{MS}, V}\left(\theta_{n, m, A o A}\right) \\
& h_{H V}=\chi_{B S, V}\left(\theta_{n, m, A o D}\right)\left(\sqrt{\frac{1}{1+\mathrm{XPD}}} \exp \left(j \Phi_{n, m}^{(h, v)}\right)\right) \chi_{M S, H}\left(\theta_{n, m, A o A}\right) \\
& h_{H H}=\chi_{B S, H}\left(\theta_{n, m, A O D}\right)\left(\sqrt{\frac{\mathrm{XPD}}{1+\mathrm{XPD}}} \exp \left(j \Phi_{n, m}^{(h, h)}\right)\right) \chi_{M S, H}\left(\theta_{n, m, A O A}\right) \\
& \beta_{\text {AoD }}=j 2 \pi \lambda^{-1}\left\{d_{s} \cos \left(\theta_{n, m, A o D}\right)\right\} \\
& \beta_{\text {AoA }}=j 2 \pi \lambda^{-1}\left\{\cos \left(\theta_{n, m, A o A}\right)\right\} \\
& \psi_{\text {AoA }}=j 2 \pi \lambda^{-1}\|\mathrm{v}\|\left[\cos \left(\theta_{n, m, A o A}-\theta_{\mathrm{v}}\right)\right]
\end{aligned}
$$

\begin{tabular}{|c|c|}
\hline Notation & Description \\
\hline$P_{n}$ & The power of the $n$th path. \\
\hline$\overline{\sigma_{S F}}$ & The lognormal shadow fading. \\
\hline$M$ & The number of subpaths per path. \\
\hline$\theta_{n, m, A o D}$ & $\begin{array}{l}\text { Absolute angle of departure (AoD) for the } m \text {-th subpath of the } n \text {-th } \\
\text { path at the BS with respect to the BS broadside. }\end{array}$ \\
\hline$\theta_{n, m, A o A}$ & $\begin{array}{l}\text { Absolute angle of arrival (AoA) for the } m \text {-th subpath of the nth pat } \\
\text { at the BS with respect to the BS broadside. }\end{array}$ \\
\hline$x_{B S, V}\left(\theta_{n, m, A o D}\right)$ & The BS antenna complex response for the V-pol. component. \\
\hline$x_{B S, H}\left(\theta_{n, m, A o D}\right)$ & The BS antenna complex response for the H-pol. component. \\
\hline$x_{M S, V}\left(\theta_{n, m, A o A}\right)$ & The antenna complex response for the V-pol. component of UE. \\
\hline$x_{M S, H}\left(\theta_{n, m, A o A}\right)$ & The antenna complex response for the H-pol. component of UE. \\
\hline$\Phi_{n, m}^{(x, y)}$ & $\begin{array}{l}\text { The random phase shift between } \mathrm{V}(\mathrm{H}) \text { of the } \mathrm{BS} \text { and } \mathrm{V}(\mathrm{H}) \\
\text { component of UE device for the } m \text {-th subpath. }\end{array}$ \\
\hline$\|\mathbf{v}\|$ & The magnitude of the velocity vector of UE. \\
\hline$\theta_{\mathbf{v}}$ & The azimuth angle of the UE velocity vector. \\
\hline$j$ & Square root of -1 . \\
\hline$d_{u}$ & $\begin{array}{l}\text { The distance from BS antenna element } s \text { form the reference }(s=1) \\
\text { antenna element in meters. For the reference antenna } s=1 \text { where } d_{1} \\
=1 \text {. }\end{array}$ \\
\hline
\end{tabular}

For equation (1)-(5), the first and the second subscript of ' $h$ ' represent the receiver (Rx) and transmitter (Tx) sides respectively. Notations of proposed DP MIMO SCM are described in Table 1.

Table 1. Notations of proposed DP MIMO SCM

The first matrix of equation (1) is rotation matrix which represents the random rotation of UE. The second channel matrix of proposed DP MIMO SCM represents the four depolarized 
propagation channels between DP transmit- and receive-antennas with XPD terms; $h_{V V}, h_{H V}$, $h_{V H}$, and $h_{H H}$.

$$
\begin{aligned}
\text { XPD } & =\frac{\text { Co-polarized receive power }}{\text { Cross-polarized receive power }} \\
& =\frac{\mathrm{E}\left\{\left|h_{V V}\right|^{2}\right\}}{\mathrm{E}\left\{\left|h_{H V}\right|^{2}\right\}}=\frac{\mathrm{E}\left\{\left|h_{H H}\right|^{2}\right\}}{\mathrm{E}\left\{\left|h_{V H}\right|^{2}\right\}}
\end{aligned}
$$

As shown in equation (9), XPD represents the polarization directivity with discrimination of the power imbalance between co-polarization and cross-polarized power components at DP receive-antenna. Therefore, low and high power leakages between two crossed received-antennas are represented by high and low XPD values respectively.

For example, the DP MIMO channel between spatially separated 32Tx DP transmit-antennas and one DP receive-antenna can be written as follows

$$
\mathbf{H}_{32 \text { Tx DP MIMO. }}=\left[\begin{array}{lllllll}
h_{1,1(\mathrm{VV})} & h_{1,2(\mathrm{VH})} & h_{1,29(\mathrm{VV})} & h_{1,30(\mathrm{VH})} & h_{1,63(\mathrm{VV})} & h_{1,64(\mathrm{VH})} \\
h_{2,1(\mathrm{HV})} & h_{2,2(\mathrm{HH})} & & h_{2,29(\mathrm{HV})} & h_{2,30(\mathrm{HH})} & h_{2,63(\mathrm{HV})} & h_{2,64(\mathrm{HH})}
\end{array}\right]
$$

where channel matrix between one collocated DP transmit- and one DP receive-antenna is represented by four depolarized propagation channels as follows

$$
\mathbf{H}_{1 \mathrm{Tx} \text { DP MIMO }}=\left[\begin{array}{ll}
h_{1,1(\mathrm{VV})} & h_{1,2(\mathrm{VH})} \\
h_{2,1(\mathrm{HV})} & h_{2,2(\mathrm{HH})}
\end{array}\right]
$$

where $h_{u, s,\left(P_{u}, P_{s}\right)}$ denotes the propagation channel between the sth transmit-antenna with $P_{s}$ polarization and the $u$ th receive-antenna with $P_{u}$ polarization.

\subsection{Proposed Single-Polarized MIMO SCM}

SP MIMO channel can be represented by odd column indexes of proposed DP MIMO SCM since odd column indexes of DP MIMO channel matrix represent the vertical SP transmit-antennas at BS. For instance, 32Tx SP MIMO channel matrix can be expressed by vertical SP transmit-antennas as follows.

$$
\mathbf{H}_{32 \text { Tx SP MIMO }}=\left[\begin{array}{lllllll}
h_{1,1(V V)} & h_{1,3(V V)} & h_{1,29(V V)} & h_{1,31(V V)} & h_{1,61(V V)} & h_{1,63(V V)} \\
h_{2,1(H V)} & h_{2,3(H V)} & \cdots & h_{2,29(H V)} & h_{2,31(H V)} & h_{2,61(H V)} & h_{2,63(H V)}
\end{array}\right]
$$

As shown in equation (12), SP MIMO channel matrix is composed of only vertical SP transmit-antennas which is represented by fourth subscript, $V$, of $h ; h_{V V}$ and $h_{H V}$ represent vertical to vertical and vertical to horizontal propagation channels between SP transmit-antennas and DP receive-antennas respectively. 


\section{Evaluation Method}

\subsection{BS-VR Length of Massive MIMO System}

BS-visibility region (BS-VR) represents the visibility of particular cluster at BS when UE enters cluster on the azimuth plane and its visible length of transmit-array is called observed BS-VR length [17]. Since the transmit-array size of massive MIMO system is much larger than the transmit-array of conventional MIMO system, the BS-VR characteristics are different from the conventional MIMO system.

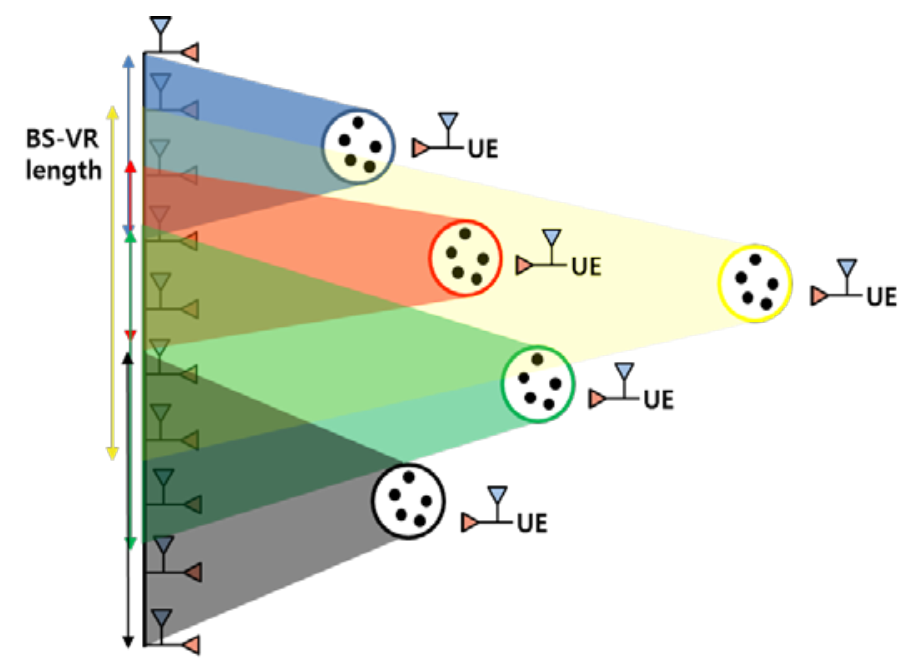

Fig. 3. Concept of BS-VR length of the massive MIMO system

As shown in Fig. 3, transmit-array of massive MIMO system has many BS-VR lengths since transmit-array is much larger than transmit-array of conventional MIMO systems. Each BS-VR length of Fig. 3 represents the transmit-array of a small MIMO system. In other words, different groups of transmit-antenna recognize the different UE clusters depending on the location of the UE for massive MIMO system whereas conventional/small MIMO system always recognizes the same clusters regardless of the length of the transmit-array.

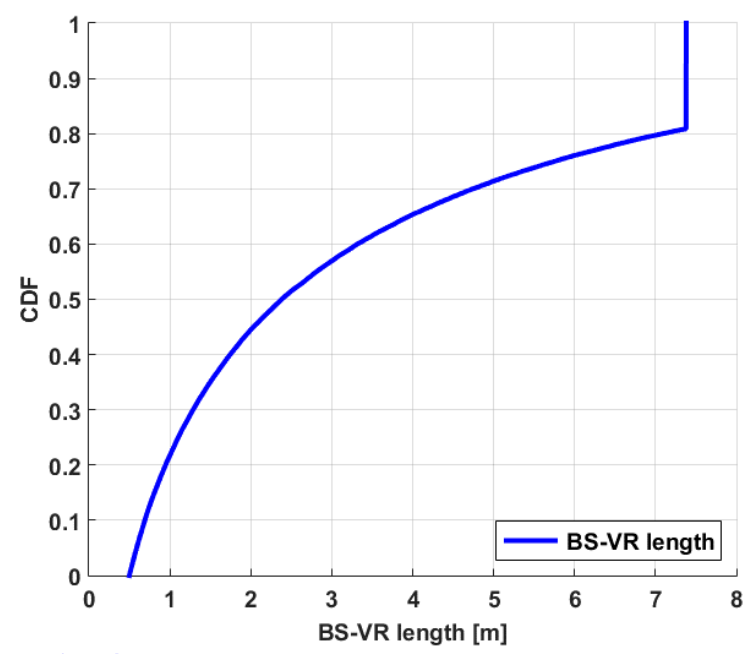

Fig. 4. BS-VR length of the massive MIMO system 
Fig. 4 shows the simulation result of the CDF of the BS-VR length from log-normal distribution with logarithm mean $\mu=0.7$ and logarithm standard deviation $\sigma=2$. The BS-VR characteristics of massive MIMO system is applied to evaluate the performance of both DP and SP massive MIMO systems since simulation results in Fig. $\mathbf{4}$ is identical as Fig. 6(b) in [17] for same simulation scenarios.

\subsection{Multi-UE Scheduling with Limited Feedback}

First of all, it is assumed that BS has perfect knowledge of AoD to serve UEs and each UE also has perfect channel state information (CSI). Since transmit-array transmits data simultaneously to optimally $S$ selected UEs among total $K$ UEs, data symbols for the $S$ selected UEs are linearly precoded by maximum ratio transmission (MRT). Then, the transmit signal vector can be written as follows

$$
\mathbf{X}=\mathbf{F} \mathbf{b}=\sum_{k=1}^{S} \mathbf{f}_{k} b_{k}
$$

where $\mathbf{F}=\left[\mathbf{f}_{1} \mathbf{f}_{2} \cdots \mathbf{f}_{S}\right]$ is the $S \times S$ precoding matrix and $\mathbf{f}_{k}$ is the $S \times 1$ precoding vector for the selected $k$ th UE devices among $S$ selected UEs. Further, $\mathbf{b}=\left[b_{1} b_{2} \cdots b_{S}\right]^{T}$ is the $S \times 1$ transmit symbol vector where $b_{k}$ is the transmit symbol for the $k$ th UE among the $S$ selected UEs.

To minimize the interference between vertical and horizontal receive-antennas, minimum mean square error (MMSE) receiver is employed at UE based on perfect knowledge of CSI. Then, the signal-to-interference-plus-noise ratio (SINR) of the $K$ th UE can be written as

$$
\gamma_{k}=b_{k}^{*} \mathbf{f}_{k}^{\mathrm{H}} \mathbf{H}_{k}^{\mathrm{H}}\left(\mathbf{I}+\sum_{l=1, l \neq k}^{M} \mathbf{H}_{k} \mathbf{f}_{l} \mathrm{~s}_{l} s_{l}^{*} \mathbf{f}_{l}^{\mathrm{H}} \mathbf{H}_{k}^{\mathrm{H}}\right)^{-1} \mathbf{H}_{k} \mathbf{f}_{k} b_{k}
$$

where $\mathbf{H}_{k}$ and $\mathbf{I}$ denote $U \times S$ DP MIMO channel of the $k$ th UE and $S \times S$ identity matrix respectively. The precoding vector of $k$ th $\mathrm{UE}$ is denoted by $\mathbf{f}_{k}$ as follows

$$
\begin{gathered}
\mathbf{f}_{k}=\mathbf{V}_{k} \\
\operatorname{SVD}\left[\mathbf{H}_{k}\right]=\mathbf{U}_{k} \boldsymbol{\Sigma}_{k} \mathbf{V}_{k}^{\mathrm{H}}
\end{gathered}
$$

where SVD denotes the singular value decomposition. $\mathbf{U}_{k}, \Sigma_{k}$ and $\mathbf{V}_{k}$ represent $S \times S$ complex unitary matrix, $S \times U$ rectangular diagonal matrix, and $U \times U$ complex unitary matrix of the $k$ th UE, respectively. Then the achievable rate of the $k$ th UE with MMSE receiver can be written as

$$
C_{k}=\log _{2}\left(1+\gamma_{k}\right)
$$

To evaluate the throughput of proposed MIMO systems, multi-UE scheduling algorithm is applied which serves multiple UE simultaneously to achieve highest UE-sum-rate among $K$ UEs by MRT precoding vectors. By MRT, the signals are transmitted by the strongest eigenmodes at BS and the received signals are combined using maximal ratio combining at UE. Then, multi-UE selection algorithm is defined as follows. 


$$
\begin{aligned}
& \text { initialize } G_{\text {opt. }}=\{\varnothing\}, C_{\max }=0, a=0 \\
& \text { do } \\
& n=\underset{n \in O, n \neq G_{\text {opt. }}}{\arg \max \left\{n, G_{\text {opt. }}\right\}} \log _{2}\left(1+\gamma_{k}\right) \\
& C=\sum_{k \in\left\{n, G_{\text {opt }}\right\}} \log _{2}\left(1+\gamma_{k}\right) \\
& \text { if } C>C_{\text {max. }} \text {. } \\
& G_{\text {opt. }}=\left\{G_{\text {opt. }}, n\right\} \\
& R_{\text {max }}=R \\
& a=1
\end{aligned}
$$

while $a=1$ and $\left|G_{o p t .}\right|<S_{t x}$

Algorithm 1. Multi-UE selection algorithm

where $O, S_{t x}, C$, and $C_{\max }$ denotes the universal set of UEs, the number of transmit-antennas, throughput, and the maximum throughput of the $k$ th UE respectively.

\subsection{System-Level Simulation}

To analyze the comparative performance between DP and SP massive MIMO systems, massive MIMO systems are evaluated by 3-sector based system-level simulation methodology [18]. Further, both vertical and horizontal transmit-antenna patterns are modeled with $3 \mathrm{~dB}$ beam width of $70^{\circ}$ with maximum gain of $17 \mathrm{dBi}$ for 3-sector macro cell scenario [16]. Moreover, in order to simplify CSI estimation for downlink channel from uplink channel, time division duplex (TDD) operation is assumed to exploit channel reciprocity [19]. The simulation parameters are presented in Table 2.

Table 2. Simulation parameters

\begin{tabular}{|l|l|}
\hline Parameter & Assumption \\
\hline \hline Cellular layout & Hexagonal grid, 19 sites, and 3 sectors per site [16] \\
\hline Simulation scenarios & Urban macro with AS $8^{\circ}$ [16] \\
\hline Sector radius & $350 \mathrm{~m}$ \\
\hline Carrier frequency & $1.9 \mathrm{GHz}$ \\
\hline System bandwidth & $10 \mathrm{MHz}$ \\
\hline Channel estimation & Ideal \\
\hline XPD & 0 and $15 \mathrm{~dB}$ \\
\hline BS height & $35 \mathrm{~m}$ \\
\hline UE height & $1.5 \mathrm{~m}$ \\
\hline Antenna spacing & $0.5 \lambda$ \\
\hline BS transmit power & $43 \mathrm{dBm}$ \\
\hline Detection algorithm at UE & MMSE \\
\hline Speed of moving UE & $3 \mathrm{~km} / \mathrm{h}$ \\
\hline Rotation of UE & Random rotation between $0^{\circ}$ and $360^{\circ}$ \\
\hline Noise figure & $7 \mathrm{~dB}$ \\
\hline Pathloss & COST 231 Hata model [20] \\
\hline
\end{tabular}




\section{Performance Analysis}

\subsection{Simulation Scenarios}

To achieve comparative performance evaluation between proposed DP and SP massive MIMO systems, three simulation scenarios are proposed; 32Tx DP, 64Tx SP, and 32Tx SP massive MIMO systems.

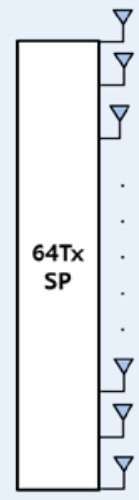

(a)

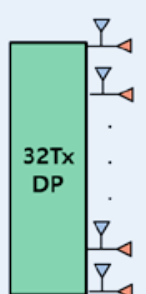

(b)

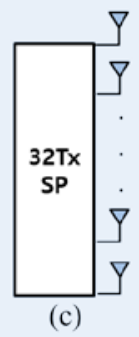

$Y:$ Single-polarized transmit-antenna

Fig. 5. Configuration of (a) 64Tx SP, (b) proposed 32Tx DP, (c) 32Tx SP massive MIMO systems

As shown in Fig. 5, 64Tx SP and 32Tx SP massive MIMO systems represent the same size and the same number of transmit-antennas as proposed 32Tx DP massive MIMO system. In other words, proposed 32Tx DP massive MIMO system has the same transmit-array size as the 32Tx SP massive MIMO system and has the same number of transmit-antennas as the 64Tx SP massive MIMO system. Furthermore, XPD values of 0 and $15 \mathrm{~dB}$ are implemented to investigate the impact of XPD for three massive MIMO systems.

\subsection{UE Throughputs of Dual- and Single-Polarized Massive MIMO Systems}

In this subchapter, UE throughputs of proposed 32Tx DP, 64Tx SP, and 32Tx SP massive MIMO systems are represented by CDF.

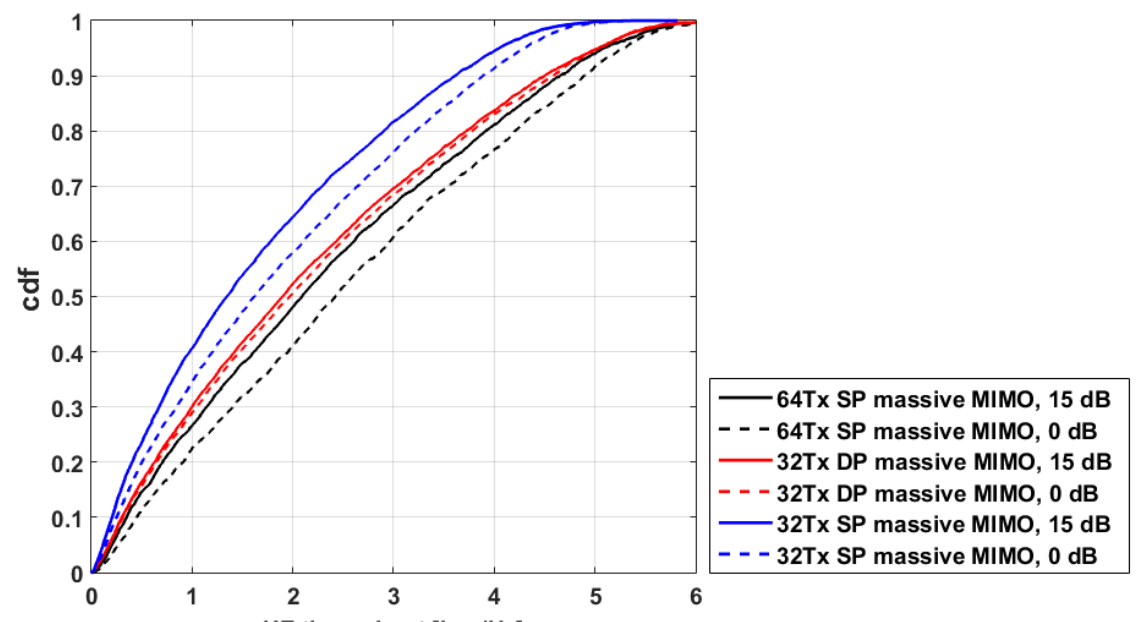

Fig. 6. UE throughputs of DP and SP massive MIMO systems. 
Fig. 6 represents the UE throughputs of proposed 32Tx DP, 64Tx SP, and 32Tx SP massive MIMO systems. As shown in Fig. 6, 64Tx and 32Tx SP massive MIMO systems perform higher UE throughput at XPD $=15 \mathrm{~dB}$ compared to $\mathrm{XPD}=0 \mathrm{~dB}$ since the higher value of XPD represents the higher directivity between vertical SP transmit-antennas and vertical receive-antennas of the DP receive-antennas.

On the other hand, DP transmit-antennas provide more diversity/selectivity for the DP receive-antenna regardless of XPD levels since vertical and horizontal transmit-antennas of each collocated DP transmit-antenna transmits two independent signals. Therefore, the difference in UE throughputs between XPD $=0 \mathrm{~dB}$ and XPD $=15 \mathrm{~dB}$ for DP massive MIMO system is less than SP massive MIMO systems. Thus, proposed 32Tx DP massive MIMO system benefits for uncorrelated channel, XPD $=0 \mathrm{~dB}$, which is advantage for conventional wireless channel.

\subsection{Five-Percentile Throughput of Dual- and Single-Polarized Massive MIMO Systems}

In this subchapter, performances of five-percentile throughputs of proposed 32Tx DP, 64Tx SP, and 32Tx SP massive MIMO systems are analyzed.

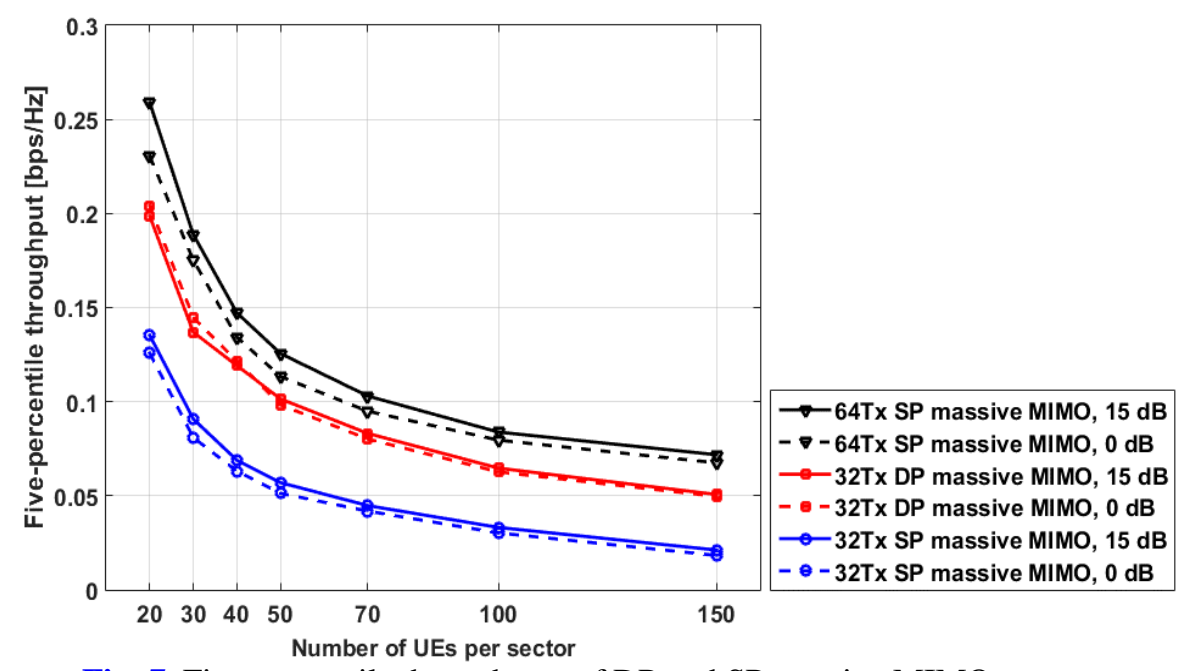

Fig. 7. Five-percentile throughputs of DP and SP massive MIMO systems.

Fig. 7 shows the five-percentile (or sector-edge) throughput of proposed 32Tx DP, 64Tx SP, and 32Tx SP massive MIMO systems. According to simulation results in Fig. 7, proposed 32Tx DP massive MIMO system performs between 64Tx SP and 32Tx SP massive MIMO systems and it performs very close to 64Tx SP massive MIMO system. This series of simulation results demonstrate that proposed 32Tx DP massive MIMO system performs very close to 64Tx SP massive MIMO system by using only half size of the 64Tx SP massive MIMO system even at the sector edge since DP transmit-antennas aids to transmit signals by extra polarization domain.

\subsection{Comparative Performance Analysis of Dual- and Single-Polarized Massive MIMO Systems}

In this subchapter, the average UE-sum-rate, average throughput in other words, of three massive MIMO systems are analyzed by system-level simulation. 


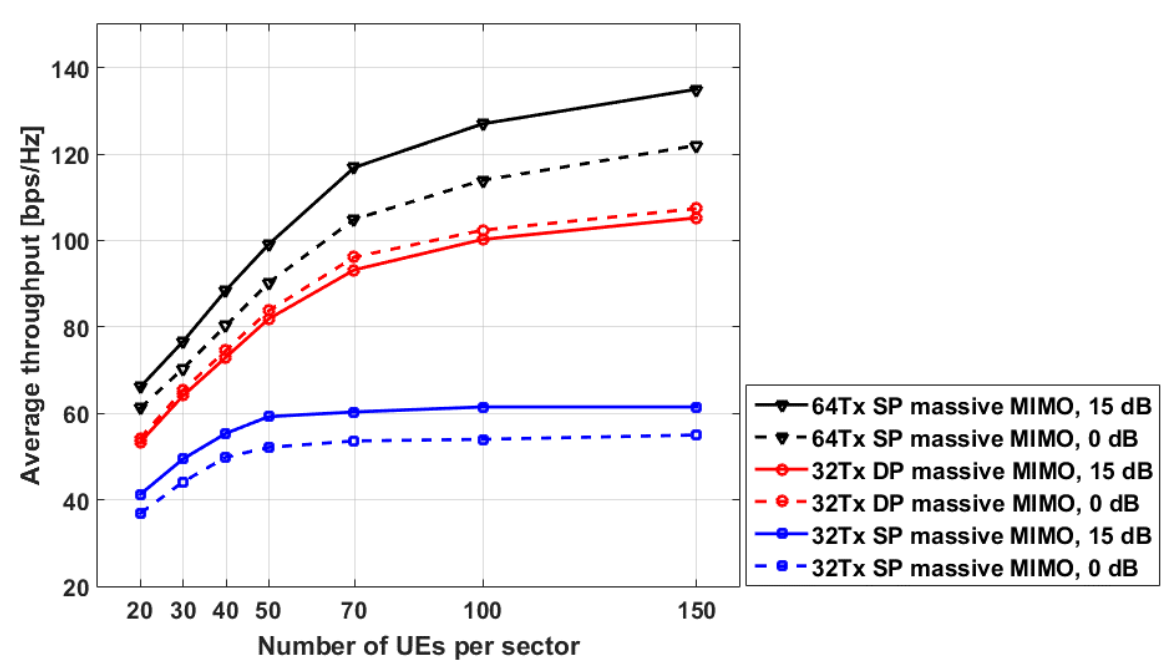

Fig. 8. Average throughputs of DP and SP massive MIMO systems.

Fig. 8. represents the comparison of average throughput of proposed 32Tx DP, 64Tx SP, and 32Tx SP massive MIMO systems as a function of number of UE. As shown in Fig. 8, proposed 32Tx DP massive MIMO system performs between 64Tx SP and 32Tx SP massive MIMO systems. Table 3 represents the performance improvement of proposed 32Tx DP massive MIMO system compared to 32Tx SP massive MIMO system for identical size of the transmit-array.

Table 3. Performance improvement of 32Tx DP massive MIMO system compared to 32Tx SP massive MIMO system

\begin{tabular}{|c|c|c|c|c|c|c|c|}
\hline & \multicolumn{7}{|c|}{ Number of UEs Per Sector } \\
\cline { 2 - 8 } & $\mathbf{2 0}$ UEs & 30 UEs & 40 UEs & 50 UEs & 70 UEs & 100 UEs & 150 UEs \\
\hline \hline XPD = 0 dB & $34.77 \%$ & $37.96 \%$ & $40.78 \%$ & $53.22 \%$ & $73.62 \%$ & $84.78 \%$ & $90.96 \%$ \\
\hline XPD = 15 dB & $16.36 \%$ & $18.95 \%$ & $22.62 \%$ & $30.08 \%$ & $47.72 \%$ & $56.98 \%$ & $65.65 \%$ \\
\hline
\end{tabular}

As shown in Table 3, the maximum performance improvement of 32Tx DP massive MIMO system is $90.96 \%$ for 150 UEs compared to 32Tx SP massive MIMO system at XPD $=0 \mathrm{~dB}$. This simulation results indicate that proposed DP massive MIMO system almost doubles the performance compared to SP massive MIMO system for a large number of UEs. Since deployment of a large number of transmit-antennas in limited space is very important for massive MIMO system, this simulation result is the most important contribution of proposed DP massive MIMO system.

\section{Conclusion}

Since a large number of transmit-antennas must be deployed for massive MIMO system, size reduction of transmit-array is very important to realize practical massive MIMO system. Therefore, DP massive MIMO system is proposed in order to deploy twice as many transmit-antennas as SP massive MIMO system. Simulation results show that the DP massive MIMO system improves performance by about $91 \%$ compared to SP massive MIMO system for the same size of the transmit-array since DP massive MIMO system utilizes space and 
polarization domains whereas SP massive MIMO system utilizes only space domain. Moreover, the performance of DP massive MIMO system is not sensitive to XPD variations which is strong advantages for both correlated and uncorrelated channel environment. Therefore, DP massive MIMO system can be the solution for next wireless communication's antenna configuration especially for massive MIMO system.

\section{References}

[1] W.-S. Jeong, S.-H. Kim, and K.-S. Min, "An analysis of the economic effects for the IoT industry,” KSII Journal of Internet Computing and Services, vol. 14, no. 5, pp. 119-128, October, 2013. Article (CrossRef Link)

H.-W. Kim and M. Lee, "Big data and entertainment content: case studies and prospects," KSII Journal of Internet Computing and Services, vol. 17, no. 7, pp.109-118, April, 2016. Article (CrossRef Link)

[2] Cisco, "Cisco visual networking index: global mobile data traffic forecast update, 2014-2019," March, 2017.

[3] A. de la Fuente, R. P. Leal, and A. G. Armada, "New technologies and trends for next generation Mobile Broadcasting Services," IEEE Commun. Mag., Vol. 54, No. 11, pp.217-223, October, 2016. Article (CrossRef Link)

[4] S.-J. Kim, "Performance analysis of fractional frequency reuse scheme for enterprise femtocell networks," KSII Journal of Internet Computing and Services, vol. 19, no. 1, pp. 11-17, February, 2018. Article (CrossRef Link)

[5] E. Larsson, O. Edfors, F. Tufvesson, and T. Marzetta, "Massive MIMO for next generation wireless systems," IEEE Commun. Mag., vol. 52, no. 2, pp. 186-195, February, 2014. Article (CrossRef Link)

[6] F. Rusek et al., "Scaling up MIMO: Opportunities and challenges with very large arrays," IEEE Signal Process. Mag., vol. 30, no. 1, pp. 40-60, January, 2013. Article (CrossRef Link)

[7] T.Marzetta, "Noncooperative cellular wireless with unlimited numbers of base station antennas," IEEE Trans. Wireless Commun., vol.9 ,no. 11, pp. 3590-3600, November, 2010. Article (CrossRef Link)

[8] J.-K. Hong, H. Joung, H.-S. Jo, C. Mun, and J.-G Yook, "A three dimensional model for dual polarized MIMO channel of the planar array antennas," International Journal of Smart Home, vol.7, no.1, January, 2013.

[9] Y.-H. Nam et al., "Full-dimension MIMO (FD-MIMO) for next generation cellular technology," IEEE Commun. Mag., vol. 51, no. 6, pp. 172-179, June, 2013. Article (CrossRef Link)

[10] W. Xu, X. Wu, X. Dong, H. Zhang, and X. You, "Dual-polarized massive MIMO systems under multi-cell pilot contamination,” IEEE Access, vol. 4, pp. 5998-6013, September, 2016. Article (CrossRef Link)

[11] J. K. Hong, H. S. Jo, C. Mun, and J. G. Yook, "Opportunistic polarization-matching for multiuser polarized MISO downlink,” IEEE Commun. Letters, vol. 21, no. 4, pp. 909-912, April, 2017. Article (CrossRef Link)

[12] S.-C. Kwon and G. Stuber, "Polarization division multiple access on NLoS wide-band wireless fading channels," IEEE Trans. Wireless Commun., vol. 13, no. 7, pp. 3726-3737, July, 2014. Article (CrossRef Link)

[13] H. Joung, H-S. Jo, C. Mun, and J-G. Yook, "Space-polarization division multiple access system with limited feedback," KSII Trans. on Internet and Information Systems, vol. 8, no. 4, pp. 1292-1306, April, 2014. Article (CrossRef Link)

[14] H. Joung, H. Jo, C. Mun, and J.-G. Yook, "Capacity loss due to polarization-mismatch and space-correlation on MISO channel," IEEE Trans. Wireless Commun., vol. 13, no. 4, pp. 2124-2136, April, 2014. Article (CrossRef Link)

[15] 3GPP TR 25.996, "Spatial channel model for multiple input multiple output (MIMO) simulations," 12.0.0 ed., 2014. 
[16] X. Gao, F. Tufvesson, and O. Edfors, "Massive MIMO channels - measurements and models," in Proc. of Asilomar Conference on Signals, Systems, and. Computers (ACSSC), Pacific Grove, CA, pp. 280-284, November, 2013. Article (CrossRef Link)

[17] R. Srinivasan, ed., "IEEE $802.16 \mathrm{~m}$ evaluation methodology document (EMD), IEEE 802.16m-advanced air interface,” January, 2009.

[18] T. L. Marzetta, "How much training is required for multiuser MIMO ?,” in Proc. of 40th Asilomar Conference on Signals, Systems, and. Computers (ACSSC), Pacific Grove, CA, pp. 359-363, November, 2006. Article (CrossRef Link)

[19] COST Action 231, "Digital mobile radio towards future generation system, final report," tech. rep., European Communities, EUR 18957, 1999.

Jun-Ki Hong was born in Seoul, South Korea. He received the B.S. in computer systems engineering from Carleton University, Ottawa, Canada, in 2010 and the Ph.D. degrees in electronics engineering from Yonsei University, Seoul, South Korea, in 2017. He joined Telecommunications Technology Association, Bundang-gu, South Korea, where he worked on developing the standardizations of V2X from 2016 to 2017. He is currently an Assistant Professor with the Department of Electrical and Electronic Engineering, Youngsan University. His current research interests include the areas of 5G wireless communications, MIMO systems, V2X, spatial channel model, and big data analysis. 